\title{
Spastic tetraplegia as an initial manifestation of familial Alzheimer's disease
}

\author{
N Sodeyama, M Shimada, T Uchihara, K Yanagisawa, H Fujigasaki, K Yamaguchi, \\ M Matsushita, M Yamada
}

Department of

Neurology, Tokyo

Medical and Dental

University, Tokyo,

Japan

N Sodeyama

T Uchihara

$\mathrm{K}$ Yanagisawa

H Fujigasaki

$M$ Yamada

Department of

Diagnostic Pathology,

Kanto Teishin

Hospital, Tokyo,

Japan

M Shimada

K Yamaguchi

Department of

Psychiatry, Faculty of

Medicine, University

of Tokyo, Tokyo,

Japan

M Matsushita

Correspondence to:

Dr N Sodeyama,

Department of Neurology,

Tokyo Medical and Dental

University, Yushima 1-5-45,

Bunkyo-ku, Tokyo 113,

Japan.

Received 9 January 1995

and in revised form

11 May 1995

Accepted 1 June 1995

\begin{abstract}
Two sisters with familial Alzheimer's disease developed spastic gait disturbance as an initial manifestation. Their gait disturbance progressed gradually, followed by dementia a few years later. Postmortem examination of one of the patients disclosed degeneration of the thalamus and corticospinal tract in addition to numerous senile plaques and neurofibrillary tangles in the neocortex, both of which were confirmed by immunohistochemistry. This is the first report in which clinicopathological evaluation is sufficient to establish a new variant of Alzheimer's disease presenting initially as spastic tetraplegia.
\end{abstract}

(F Neurol Neurosurg Psychiatry 1995;59:395-399)

Keywords: familial Alzheimer's disease; spastic tetraplegia; thalamic degeneration

Alzheimer's disease is a degenerative disorder of the brain characterised clinically by progressive dementia and pathologically by senile plaques and neurofibrillary tangles. ${ }^{12}$ Although some cases with unusual clinicopathological features have been classified as atypical Alzheimer's disease, such diagnoses are uncertain. ${ }^{34}$ We describe the clinicopathological findings in two siblings who developed progressive spastic tetraplegia followed by dementia. Immunohistochemical examinations confirmed the diagnosis of Alzheimer's disease.
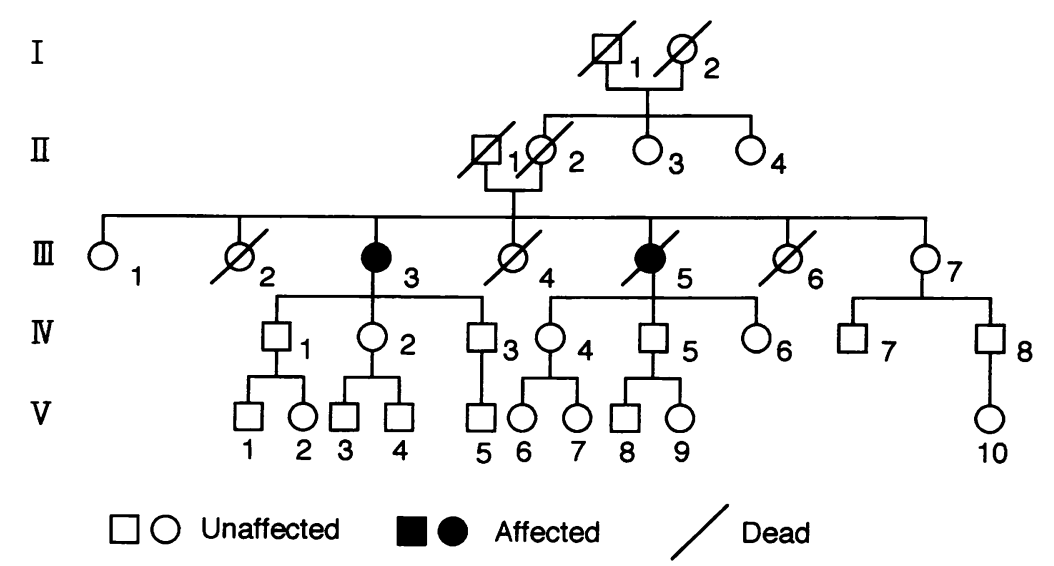

Figure 1 Family pedigree.

\section{Case reports}

PATIENT 1 (III-5, FIG 1)

Patient 1 had non-consanguineous parents. Her history disclosed a fracture of the fifth lumbar vertebra in a traffic accident at the age of 51, and standard radical mastectomy for left breast cancer at the age of 58 . Neither was associated with neurological sequelae. Her first neurological symptom was spastic gait disturbance at the age of 57. At the age of 60 , memory impairment and decreased mental activities in daily life were noted. She also showed difficulty in dressing and using instruments of daily life due to spastic paresis in her upper limbs. Her spastic tetraplegia and dementia progressed gradually. At the age of 64 , she became bedridden and showed frequent nocturnal delirium and emotional incontinence. On admission, she was alert but disoriented for time and place. She correctly named only two of 10 objects presented. She answered with only a single word to some simple questions. She could not repeat a short sentence. Her score on the Mini mental state scale was 4. Cranial nerves were normal except for mild dysarthria. Weakness, atrophy, postural tremor, hyperreflexia, and muscle contracture in all limbs, and bilateral Babinski's signs were noted. There was neither sensory deficit nor cerebellar signs. She was incontinent of urine and faeces. Blood, urine, and CSF examinations were unremarkable. An EEG showed a moderate degree of generalised slowing. An EMG and nerve conduction study were normal. Brain MRI showed moderate frontotemporal atrophy (fig 2A). Single photon emission computed tomography (SPECT) showed diffuse cerebral hypoperfusion. Cervical spinal cord MRI was unremarkable except for mild spinal canal stenosis. She was diagnosed as having atypical Alzheimer's disease. During the next six months her general condition deteriorated further with decubitus ulcers and pneumonia. At the age of 65 , she died of empyema. The general necropsy showed empyema, multiple erosion of the gastric mucosa, atrophy of the pancreas, small stones in the bladder, myoma uteri, and an adenoma in the thyroid.

\section{Gross neuropathology}

The weight of the unfixed brain was $1030 \mathrm{~g}$. The brain showed no abnormalities externally. Coronal sections of the cerebrum, cerebellum, and brainstem were unremarkable. Purulent pachymeningitis at the lumbar level was noted. Transverse sections of the spinal cord showed no abnormalities. 

weighted MRI of the brain of patient 1 showing moderate frontotemporal atrophy. (B) Plain CT of the brain of patient 2 showing the same distribution pattern of atrophy as in patient 1.
Figure 2 (A) Axial T1
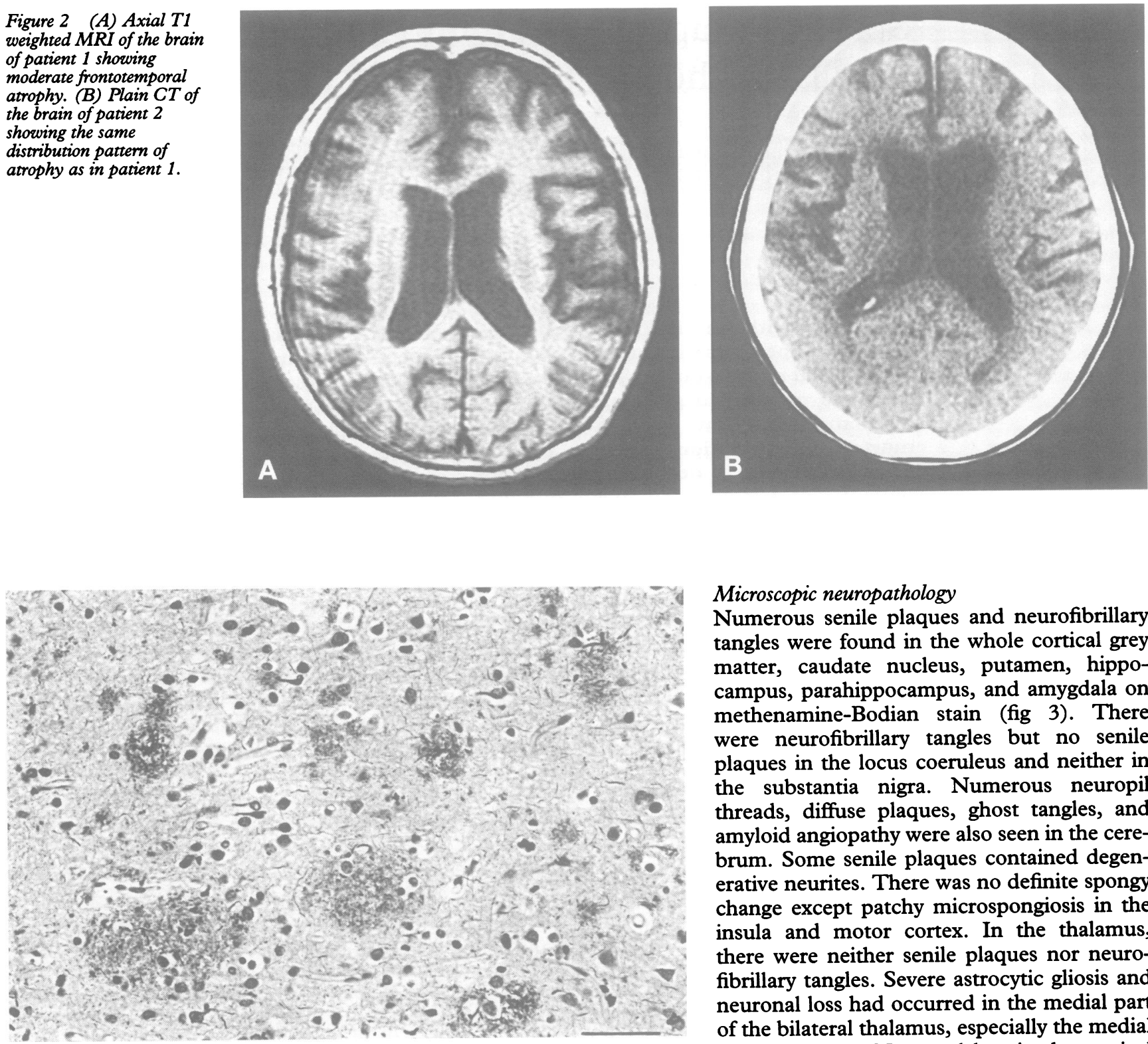

Figure 3 Numerous senile plaques and neurofibrillary tangles in the temporal cortex; methenamine-Bodian stain; magnification originally $\times 80 ;$ bar $=50 \mu \mathrm{m}$.

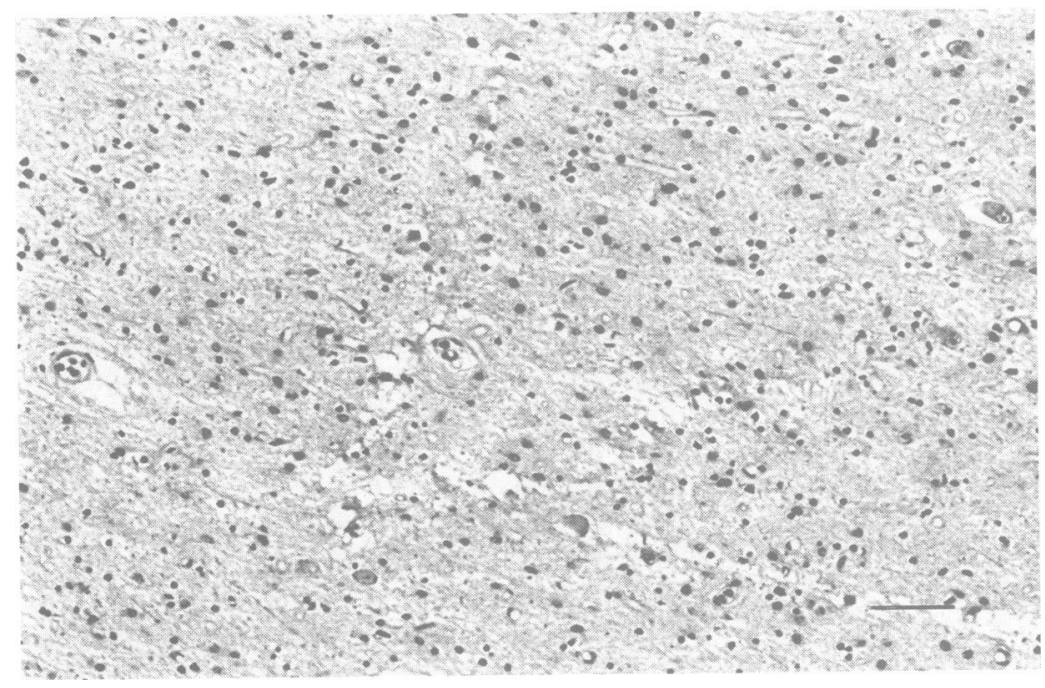

Figure 4 Section of the medial nucleus of the thalamus showing severe astrocytic gliosis and neuronal loss; haematoxylin-eosin stain; magnification originally $\times 50 ;$ bar $=50 \mu \mathrm{m}$.

\section{Microscopic neuropathology}

Numerous senile plaques and neurofibrillary tangles were found in the whole cortical grey matter, caudate nucleus, putamen, hippocampus, parahippocampus, and amygdala on methenamine-Bodian stain (fig 3). There were neurofibrillary tangles but no senile plaques in the locus coeruleus and neither in the substantia nigra. Numerous neuropil threads, diffuse plaques, ghost tangles, and amyloid angiopathy were also seen in the cerebrum. Some senile plaques contained degenerative neurites. There was no definite spongy change except patchy microspongiosis in the insula and motor cortex. In the thalamus, there were neither senile plaques nor neurofibrillary tangles. Severe astrocytic gliosis and neuronal loss had occurred in the medial part of the bilateral thalamus, especially the medial nucleus (fig 4). Neuronal loss in the cortical grey matter, amygdala, locus coeruleus, basal nucleus of Meynert, putamen, caudate nucleus, and hippocampus was mild. Neurons in the substantia nigra were preserved. The cerebral white matter was normal. There were no senile plaques or neurofibrillary tangles in the cerebellum. Betz cells of the motor cortex, the internal capsule, and pyramidal tract in the brainstem were preserved (fig 5A). Neuronal loss, mild astrocytic gliosis, and slight spongiform change, however, were found in the external granular layer of the motor cortex (fig 5B). The corticospinal tract showed pronounced symmetric pallor at all levels of the spinal cord (fig 6). A similar change was found in the bilateral anterior funiculus. Anterior horn cells were intact except for mild gliosis and some central chromatolysis.

To characterise senile plaques and neurofibrillary tangles, immunohistochemical studies using antibodies to amyloid $\beta$ protein, ${ }^{5}$ human tau protein, ${ }^{6}$ and prion protein ${ }^{7}$ were performed as previously described. ${ }^{78}$ Senile plaques and the walls of the vessels were immunostained by antibody to amyloid $\beta$ 
Figure 5 Section of the motor cortex. (A) Betz cells were well preserved. The amount of lipofuscin deposited in the cytoplasm of Betz cells is consistent with the patient's age; haematoxylin-eosin stain; magnification originally $\times 50$; bar $=50 \mu \mathrm{m}$. (B) Neuronal loss, mild astrocytic gliosis, and slight spongiform change in the external granular layer are shown; haematoxylin-eosin stain; magnification originally $\times 20$ bar $=200 \mu \mathrm{m}$

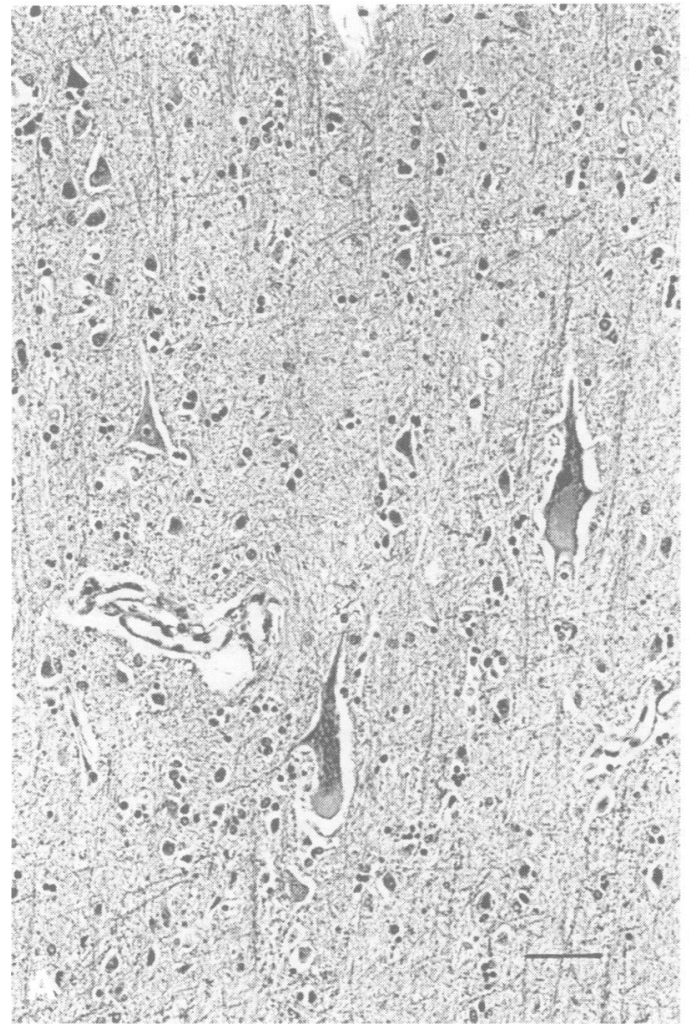

protein, but not by antibody to prion protein. Degenerative neurites of senile plaques and neurofibrillary tangles were labelled by antibody to human tau protein. Western blot analysis of the brain tissue excluded the presence of an abnormal prion protein. ${ }^{7}$

PATIENT 2 (III-3, FIG 1)

The elder sister of patient 1 developed spastic gait disturbance at the age of 53. At the age of 59, memory impairment, lack of initiation, and deterioration in homemaking performance were noted. Neurological evaluation at the age of 60 disclosed remarkable spasticity

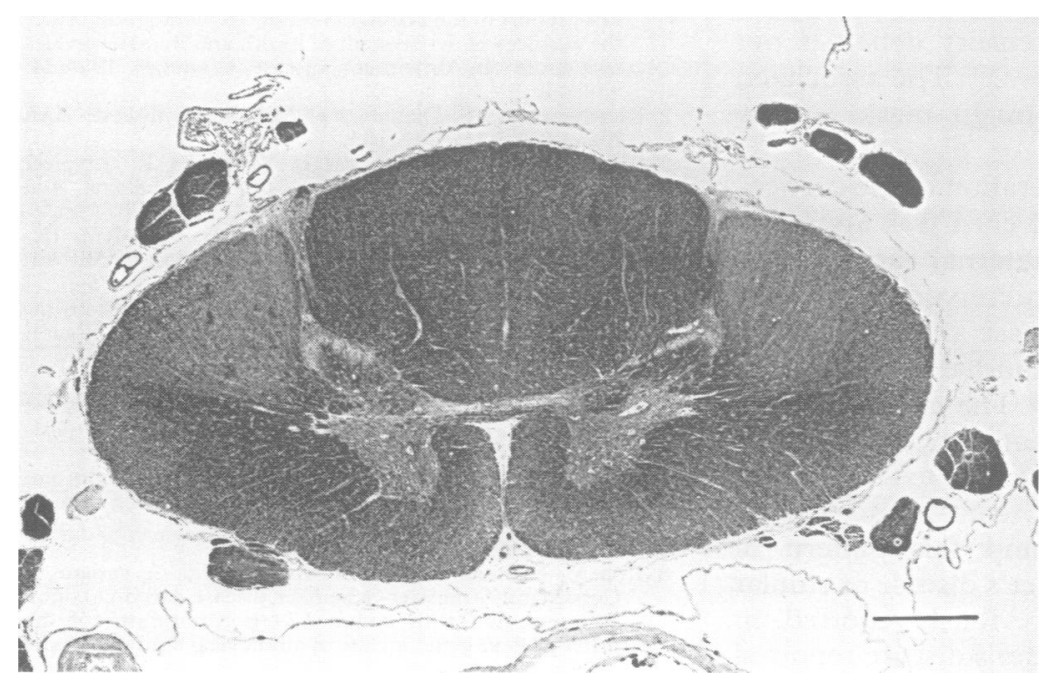

Figure 6 Section of the thoracic spinal cord showing symmetric pallor of the corticospinal tract and anterior funiculus; Klüver-Barrera stain; magnification originally $\times 3 \cdot 3$; bar $=1 \mu \mathrm{m}$.

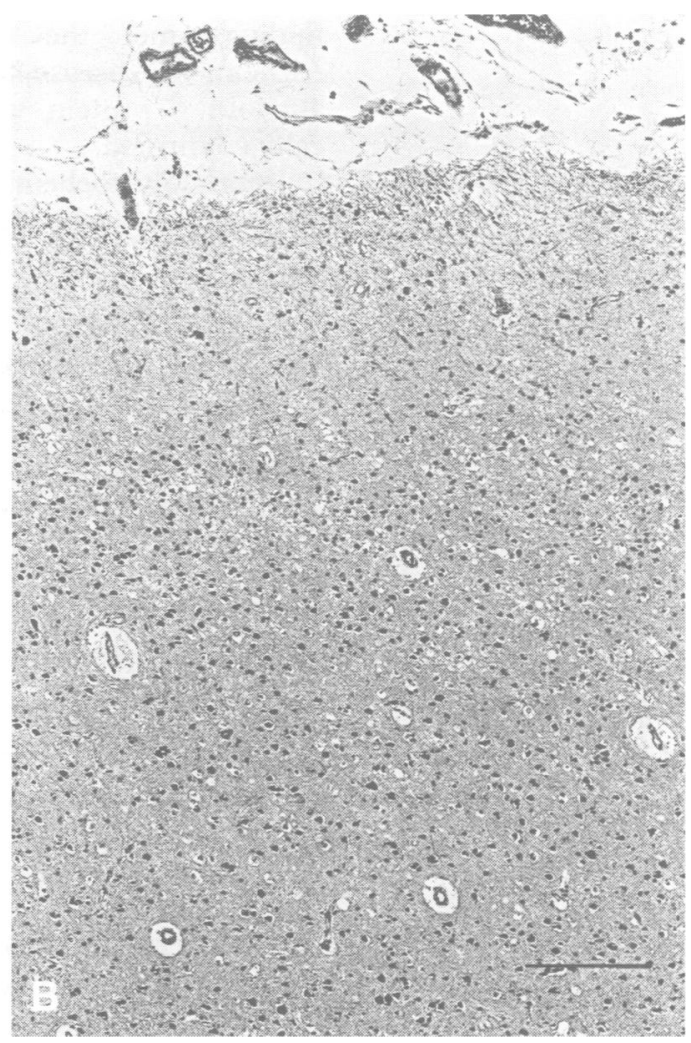

ower extremities, diffuse hyperreflexia, bilateral Babinski's sign, and dementia (a total IQ on the WAIS was 65). No sensory deficit was detected. Laboratory investigation was unremarkable and EEG, EMG, and a nerve conduction study were normal. Brain CT showed moderate frontotemporal atrophy (fig 2B). Her neurological symptoms worsened gradually. At the age of 64, she became bedridden. She could follow only simple commands and showed frequent emotional incontinence. On neurological examination at the age of 67 , she had lost all ability to communicate. She followed objects with her eyes and responded to her name. She mumbled incomprehensible words and could not follow any commands. Cranial nerves were normal except for mild dysarthria. She lost the ability of voluntary movement of all limbs, which showed severe contracture and muscle atrophy.

There was no similar disease in other members in our patients' pedigree (fig 1). HTLV-1 antibody was not detected in either patient. Sequencing analysis of the prion protein gene open reading frame in both patients and exons 16 and 17 of the amyloid $\beta$ protein precursor gene in patient 2 showed no mutation. ${ }^{910}$

\section{Discussion}

Our patients were clinically characterised by familial occurrence of progressive spastic tetraplegia before dementia developing in their $50 \mathrm{~s}$, and pathologically by degeneration of the thalamus and corticospinal tracts. Although clinicopathological features in our patients are far from those of typical Alzheimer's disease, ${ }^{12}$ the neuropathological 
findings met the diagnostic criteria for Alzheimer's disease, ${ }^{2}$ and the deposition of amyloid $\beta$ protein was immunohistochemically confirmed.

Immunohistochemical findings also clearly differentiated the disease affecting our patients from some variants of familial prion disease that show development of dementia with degeneration of the corticospinal tract, ${ }^{8911-13}$ or motor disturbances and dementia with thalamic degeneration. ${ }^{14}{ }^{15}$ Because the SPECT study of severely affected patients with Alzheimer's disease often shows diffuse cerebral hypoperfusion, ${ }^{16}{ }^{17}$ the SPECT findings obtained for patient 1 do not conflict with the diagnosis of Alzheimer's disease. Therefore, the disease affecting our patients could be placed within the range of Alzheimer's disease.

Pyramidal signs are rarely noted in Alzheimer's disease. Hyperreflexia was found in $18.5 \%$ of patients with Alzheimer's disease, and Babinski's signs in $2 \cdot 7 \% .^{18}$ Severe spastic tetraplegia early in the disease is exceptionally rare in Alzheimer's disease. ${ }^{1}$ Moreover, Alzheimer's disease with spastic paresis is generally considered to occur in younger persons than classic Alzheimer's disease, and many patients with Alzheimer's disease showing spastic paresis reported in the medical literature have cerebellar signs; different from our patients' clinical findings. ${ }^{3}$

Thalamic degeneration with spastic paresis was reported in thalamic dementia associated with motor neuron disease, ${ }^{19}$ familial multiple system atrophy, ${ }^{20}$ fatal familial insomnia, ${ }^{15}$ and primary degeneration of the thalamus. ${ }^{21}{ }^{22}$ Pronounced degeneration of the medial thalamus in familial juvenile Alzheimer's disease with spastic paresis was reported by Fukutani et al. ${ }^{23}$ Although their patient and ours showed degeneration of the bilateral pyramidal tracts in the distal spinal cord, considerable myelin loss and axonal damage in the cerebral white matter of their patient were not found in ours. Both patients in our study developed emotional incontinence and dementia without sensory disturbance. Although delirium and dementia are often noted in cases of bilateral thalamic degeneration, sensory deficit is not found in many such cases. ${ }^{18-22}$ The associated delirium and dementia might mask sensory deficit.

In our patients, degeneration of the corticospinal tract in the spinal cord was apparent, whereas Betz cells, the internal capsule, and anterior horn cells were preserved as found in atypical Alzheimer's disease associated with motor neuron disease reported by Matsuoka et $a l^{24}$ and Barrett et al. ${ }^{25}$ The causal relation of neuronal loss, mild astrocytic gliosis, and slight spongiform change in the external granular layer of the motor cortex and spastic tetraplegia is unclear, and this pattern of abnormalities in Alzheimer's disease or similar disorders has not been previously reported. In a case of atypical Alzheimer's disease reported by Aikawa et $a l{ }^{3}$ degeneration of the corticospinal tract spread to the cerebral white matter, brainstem, and spinal cord.
Cases of Alzheimer's disease or similar disorders associated with spastic paralysis have been reported by some authors, including Fukutani et al, Matsuoka et al, Barrett et al, and Aikawa et al, as mentioned above. ${ }^{323-30}$ In these patients, however, the evidence is insufficient to make a clear diagnosis of Alzheimer's disease. No case of this kind should be categorised as atypical Alzheimer's disease unless both the presence of amyloid plaques containing amyloid $\beta$ protein and the absence of prion disease are confirmed immunohistochemically. ${ }^{4}$

The changes in the thalamus and corticospinal tract in our patients were sufficiently severe that they could be considered not to be secondary effects of degeneration of other structures. The neuropathological findings of patient 1-namely, the combination of the corticospinal tract and thalamic degeneration, and the presence of senile plaques and neurofibrillary tangles-well explain the clinical features of the case. The presence of similar clinical features in her sibling (patient 2) suggests that the combination of neuropathological findings is hardly explained by chance. Therefore all clinicopathological findings in our patients should originate from familial Alzheimer's disease.

Alzheimer's disease is generally thought to represent several different biological disorders. ${ }^{31}$ Spastic tetraplegia of presenile onset cannot be sufficient evidence to exclude the diagnosis of this type of Alzheimer's disease. This is the first report in which clinicopathological evaluation is sufficient to establish a new variant of familial Alzheimer's disease showing spastic tetraplegia.

We thank Dr Hiroshi Mori for providing antibodies to amyloid $\beta$ protein and human tau protein, Dr Tetsuyuki Kitamoto for $\beta$ protein and human tau protein, Dr Tetsuyuki Kitamoto for confirming by immunoblot and immunohistochemistry the Kaneko for his support.

1 Mckhann G, Drachman D, Folstein M, Katzman R, Price $D$, Stadlan EM. Clinical diagnosis of Alzheimer's disease: report of the NINCDS-ADRDA work group under the auspices of department of health and human services the force on Alzheimer's disease. Neurology 1984;34: task -44 .

2 Khachaturian ZS. Diagnosis of Alzheimer's disease. Arch Neurol 1985;42:1097-105.

3 Aikawa $H$, Suzuki K, Iwasaki Y, Iizuka R. Atypical Alzheimer's disease with spastic paresis and ataxia. $A n n$ Neurol 1985;17:297-300.

4 Tomlinson BE. Aging and the dementia. In: Adams JH Duchen LW, eds. Greenfield's neuropathology. 5th ed. London: Edward Arnold, 1992:1284 410.

5 Mori H, Takio K, Ogawara M, Selkoe DJ. Mass spectrometry of purified amyloid $\beta$ protein in Alzheimer's disease. f Biol Chem 1992;267:17082-6.

6 Endoh R, Ogawara M, Iwatsubo T, Nakano I, Mori H. Lack of the carboxyl terminal sequence of tau in ghost tangles of Alzheimer's disease. Brain Res 1993;601: tangles

7 Kitamoto T, Tateishi J. Immunohistochemical confirmation of Creutzfeldt-Jakob disease with a long clinical course with amyloid plaque core antibodies. Am $\mathcal{F}$ Pathol course with amyloid

8 Itoh Y, Yamada $\dot{M}$, Hayakawa $M$, et al. A variant of Gerstmann-Sträussler-Scheinker disease carrying codon 105 mutation with codon 129 polymorphism of the prion protein gene: a clinicopathological study. $\mathcal{f}$ Neurol Sci (in press)

9 Yamada M, Itoh Y, Fujigasaki H, et al. A missense mutation at codon 105 with codon 129 polymorphism of the prion protein gene in a new variant of Gerstmann-SträusslerScheinker disease. Neurology 1993;43:2723-4. 
10 Fujigasaki H, Naruse S, Kaneko K, Hirasawa H, Tsuji S, Miyatake T. Mutational analysis of the amyloid precursor protein gene in Japanese familial Alzheimer's disease protein gene in Japanese familial A
kindreds. Hum Genet 1994;93:460-2.

11 Nakazato Y, Ohno R, Negishi T, Hamaguchi K, Arai E. An autopsy case of Gerstmann-Sträussler-Scheinker's disease with spastic paraplegia as its principle feature. Clinical Neurology (Tokyo) 1991;31:987-92. (In Japanese.)

12 Kitamoto T, Amano N, Terao Y, et al. A new inherited prion disease (PrP-P105L mutation) showing spastic paraparesis. Ann Neurol 1993;34:808-13.

13 Palmer MS, Sidle KCL, Campbell TA, Roberts GW, Plant G, Collinge J. Absence of PrP gene mutation in patient showing $\operatorname{PrP}$ immunostaining [abstract]. Neurobiol Aging 1992;13:S94.

14 Medori R, Tritschler HJ, LeBlanc A, et al. Fatal familial insomnia, a prion disease with a mutation at codon 178 of the prion protein gene. $N$ Engl $₹$ Med 1992;326:444-9.

15 Manetto V, Medori R, Cortelli P, et al. Fatal familial insomnia: clinical and pathologic study of five new cases. Neurology 1992;42:312-9.

16 O'Brien JT, Eagger S, Syed GMS, Sahakian BJ, Levy R. A study of regional cerebral blood flow and cognitive performance in the Alzheimer's disease. $\mathcal{F}$ Neurol Neurosurg Psychiatry 1992;55:1182-7.

17 Waldemar G, Bruhn P, Kristensen M, Johnsen A, Paulson $O B$, Lassen NA. Heterogeneity of neocortical cerebral blood flow deficits in dementia of Alzheimer type: a [99mT c]-d, 1-HMPAO SPECT study. $\mathcal{f}$ Neurol Neurosurg Psychiatry 1994;57:285-95.

18 Chui HC, Teng EL, Henderson VW, Moy AC. Clinical subtypes of dementia of the Alzheimer type. Neurology 1985;35:1544-50.

19 Deymeer F, Smith TW, DeGirolami U, Drachman DA. Thalamic dementia and motor neuron disease. Neurology 1989;39:58-61.

20 Katz DA, Naseem A, Horoupian DS, Rothner AD, Davies P. Familial multisystem atrophy with possible thalamic dementia. Neurology 1984;34:1213-7.
21 Hori A, Ikeda K, Kosaka K, Shinohara S, Iizuka R. System degeneration of the thalamus. Archiv für Psychiatrie und degeneration of the thalamus. Archiveiten 1981;231:71-80.

22 Oda M, Yoshimura T, Okumura A. Degeneration of the central nervous system enhanced in the thalamus. Shinkei Kenyu no Shinpo 1973;17:238-55. (In Japanese.)

23 Fukutani Y, Nakamura I, Kobayashi K, Yamaguchi N, Matsubara R. An autopsy case of familial juvenile Alzheimer's disease with extensive involvement of the subcortical grey and white matters. Acta Neuropathol 1989;77:329-32.

24 Matsuoka T, Miyoshi K, Saka $\mathrm{K}$, et al. A case of encephalopathy with plaque-like bodies, neurofibrillary change, angiopathy and amyotrophic lateral sclerosislike lesions. Shinkei Kenyu no Shinpo 1967;11: 801-11. (In Japanese.)

25 Barrett AM. A case of Alzheimer's disease with unusual neurological disturbances. $\mathcal{F}$ Nerv Ment Dis 1913;40:

26 Jervis GA, Soltz SE. Alzheimer's disease-the so-called juvenile type. Am $\mathcal{F}$ Psychiatry 1936;93:39-56.

27 McMenemey WH, Worster-Drought C, Flind J, Williams HG. Familial presenile dementia: report of case with clinical and pathological features of Alzheimer's disease. fournal of Neurology and Psychiatry 1939;2:293-302.

28 Ishino $H$, Higashi $S$, Chûta $M$, Ohta $H$. A case of Alzheimer's disease with myoclonus: amyloid plaques and grumose alteration in the cerebellum. Clinical Neurology (Tokyo) 1983;23:577-84. (In Japanese.)

29 Ferrer I, Aymami A, Rovira A, Veciana JMG. Growth of abnormal neurites in atypical Alzheimer's disease: a study with the Golgi method. Acta Neuropathol 1983; 59:167-70.

30 Friede RL, Magee KR. Alzheimer's disease: presentation of a case with pathologic and enzymatic-histochemical of a case with pathologic and enzymatic-

31 Bird TD, Sumi SM, Nemens EJ, et al. Phenotypic heterogeneity in familial Alzheimer's disease: a study of 24 kindreds. Ann Neurol 1989;25:12-25. 